\title{
The benefits of adversarial collaboration for commentaries
}

To the Editor - Most academic journals offer scholars the opportunity to comment on recently published articles. Commentaries might, for instance, describe opposing viewpoints, provide critical reappraisals or add new empirical evidence that contradicts the conclusions of the original article. Commentaries ought to be considered a source of novel insights and an important component of scientific self-correction ${ }^{1}$, yet they are often perceived as pointless quibbles ${ }^{2}$. To improve upon this situation, we advocate for commentaries based on adversarial collaboration, in which commenters and the original authors work together to draft a consensus statement. As the resulting contribution is clearer, endorsed by all parties and free of ad hominem attacks, it will arguably have a more substantial impact on the field.

The traditional commentary-plus-reply format leaves a lot to be desired, in that authors often talk past each other or even engage in hostile back-and-forths. As a reader, it can be difficult to distil a take-home message from such exchanges. They might also create or feed into the perception that scientific conclusions are merely opinions that one can disagree about. In contrast, the core philosophy of commentaries based on adversarial collaboration is cooperation and progress rather than opposition or competition. Inspired by the idea of adversarial collaborations ${ }^{3}$-in which researchers with opposing convictions collaborate on studies designed to settle their differencescommentaries based on adversarial collaboration seek to bring critics of an article and the original authors together. The end goal is a jointly authored commentary that describes a newly found, common understanding or, at the least, clearly conveys where both parties' views converge and diverge. In the latter case, it ideally also explains how the matter could be resolved, for instance, by agreeing on a critical experiment, which, in turn, could be jointly pursued (see refs. ${ }^{4,5}$ for concrete examples).

Commentaries based on adversarial collaboration can also address issues that arise during the review process for traditional commentaries and replies. In the latter system, involving the original authors as reviewers is often at the discretion of the editor. Similarly, there are no widely agreed-upon standards for replies to commentaries. Are the commentators invited for review? Are the reviewers for the commentary and reply the same or not? Furthermore, in cases where no reply gets published, it is often unclear why. Did the authors of the original article endorse the content of the commentary, were they not involved in the process, did they not care to write a reply or was their reply not accepted for reasons unknown?

The adversarial collaborative commentary model circumvents those issues, as it involves working together rather than evaluating each other, and the end-product is a mutually agreed-upon, clear-cut message to the (scientific) community. Of course, adversarial collaborative commentaries are no silver bullet to solve all disagreements.
This model should not discourage scholarly discussion, and care should be taken that valuable contributions do not get (self-) censored. This situation might arise when there is an imbalance between the parties involved (e.g., early-career researcher versus well-established, tenured professor), hence there is a certain burden on journal editors to act as a neutral arbiter; alternatively, editors could appoint an impartial expert to oversee the process. Yet even though it might require some additional effort, the benefits outlined here surely outweigh the costs.

\section{Tom Heyman (D) $1 \times$, Pieter Moors (D) 2 and Hugh Rabagliati (D) 3 \\ ${ }^{1}$ Methodology and Statistics Unit, Institute of Psychology, Leiden University, Leiden, The Netherlands. ${ }^{2}$ Laboratory of Experimental Psychology, Department of Brain and Cognition, KU Leuven, Leuven, Belgium. ${ }^{3}$ School of Philosophy, Psychology \& Language Sciences, University of Edinburgh, Edinburgh, UK.

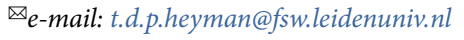

Published online: 26 October 2020 https://doi.org/10.1038/s41562-020-00978-6

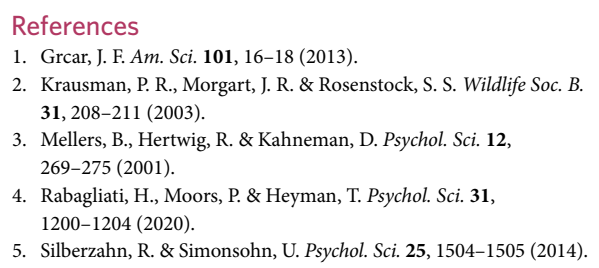

Competing interests

The authors declare no competing interests. 